\title{
Mosaic maternal 10qter deletions are associated with FRA10B expansions and may cause false-positive noninvasive prenatal screening results
}

\author{
Karin Huijsdens-van Amsterdam, $\mathrm{PhD}^{1,5}$, Roy Straver, $\mathrm{MSc}^{2,5}$, Merel C. van Maarle, MD, PhD ${ }^{1}$, \\ Alida C. Knegt, $\mathrm{PhD}^{1}$, Diane Van Opstal, $\mathrm{PhD}^{3}$, Frank Sleutels, $\mathrm{PhD}^{3}$, Dominique Smeets, $\mathrm{PhD}^{4}$ and \\ Erik A. Sistermans, $\mathrm{PhD}^{2}$
}

Purpose: Using genome-wide noninvasive prenatal screening (NIPS), we detected a 20-megabase specific deletion starting at 10q25 in eight pregnancies. The deletion could not be confirmed by invasive testing. Since all 10 (q25 $\rightarrow$ qter) deletions started close to the FRA10B fragile site in 10q25, we investigated whether the pregnant women were indeed carriers of FRA10B.

Methods: We performed NIPS analysis for all autosomes using single-read sequencing. Analysis was done with the WISECONDOR algorithm. Culture of blood lymphocytes with bromodeoxyuridine was used to detect FRA10B expansions. Fluorescence in situ hybridization and array analysis were used to find maternal and/or fetal deletions.

Results: We confirmed the presence of a FRA10B expansion in all four tested mothers. Fluorescence in situ hybridization and array analysis confirmed the presence of a maternal mosaic deletion of 10 (q25 $\rightarrow$ qter).

Conclusion: The recurring 10 (q25 $\rightarrow$ qter) deletion detected with NIPS is a false-positive result caused by a maternal low-level mosaic deletion associated with FRA10B expansions. This has important consequences for clinical follow-up, as invasive procedures are unnecessary. Expanded maternal FRA10B repeats should be added to the growing group of variants in the maternal genome that may cause false-positive NIPS results.

Genet Med advance online publication 1 March 2018

Key Words: false-positive results; FRA10B; fragile sites; NIPS; noninvasive prenatal screening

\section{INTRODUCTION}

Noninvasive prenatal screening (NIPS) is now used worldwide for the detection of common aneuploidies. With NIPS, cell-free fetal DNA in the maternal plasma is analyzed. Even though NIPS is a highly sensitive and specific screening test, false-positive results are sometimes obtained. Most falsepositive results can be explained by confined placental mosaicism, since the primary source of fetal DNA in the maternal circulation is the placental cytotrophoblast. ${ }^{1}$ Other causes of false-positive NIPS results are maternal mosaicism, ${ }^{2}$ maternal copy-number variants, ${ }^{3}$ maternal cancer, $^{4}$ or a vanishing twin. ${ }^{5}$

We detected a roughly 20 megabase $(\mathrm{Mb})$ deletion of 10 (q25 $\rightarrow$ qter) in eight independent samples tested. During extensive follow-up investigations, the detected loss on chromosome 10 was initially not seen in fetal, maternal, or placental tissue. The deletions showed strong similarities in breakpoint locations, while sample analyses were performed in three different centers and at different points in time, thus ruling out run- and laboratory-specific effects as a cause. In an effort to explain these false-positive NIPS results, it was noticed that the proximal breakpoint of the deletion occurred at the location of FRA10B. As it is known that expression of fragile sites is associated with mosaic deletions, we questioned whether this fragile site could be involved in the apparent NIPS deletion. ${ }^{6}$

Fragile sites are heritable specific chromosome loci that exhibit an increased frequency of gaps, poor staining, constrictions, or breaks when chromosomes are exposed to partial DNA replication inhibition. They are classified as common (present in all individuals) or rare $(<5 \%$ of the population) and are further subdivided into different groups based on their specific induction chemistry. ${ }^{7}$ There are two known fragile sites for 10q25: FRA10B and FRA10E. FRA10E is a common fragile site and thus present in all (or nearly all) individuals. In contrast, FRA10B is a rare and non-folatesensitive fragile site. Although it classifies as rare, the population frequency of cytogenetic expression of FRA10B is $\sim 1 / 40$ in the Australian population. ${ }^{8}$ In the group of nonfolate-sensitive fragile sites, FRA10B, together with FRA12C, is unique in that it requires bromodeoxyuridine (BrdU) for expression, whereas it is not inducible by AT-minor groove

\footnotetext{
${ }^{1}$ Department of Clinical Genetics, Academic Medical Center, Amsterdam, The Netherlands; ${ }^{2}$ Department of Clinical Genetics, VU University Medical Center Amsterdam, Amsterdam, The Netherlands; ${ }^{3}$ Department of Clinical Genetics, Erasmus Medical Center, Rotterdam, The Netherlands; ${ }^{4}$ Department of Genetics, Radboud University Nijmegen Medical Centre, Nijmegen, The Netherlands. Correspondence: Erik A. Sistermans (e.sistermans@vumc.nl)

${ }^{5}$ The first two authors contributed equally to this work.

Submitted 6 November 2017; accepted 26 January 2018; advance online publication 1 March 2018. doi:10.1038/gim.2018.32
} 
binders such as distamycin A. ${ }^{7}$ The core of FRA10B consists of a variety of AT-rich (91\%) repeats. These AT-rich repeats vary in size between 16 and 52 base pairs, at least 16 different alleles have been described. ${ }^{9}$ Normal alleles can be divided into three groups: small normal (repeat $<1$ kilobases $(\mathrm{kb})$; $66 \%$ of normal alleles), intermediate normal (repeat $1-2 \mathrm{~kb}$; $33 \%$ of normal alleles), and long normal $(2-5 \mathrm{~kb} ; 1 \%$ of normal alleles). Expanded repeats are $>5 \mathrm{~kb}$ in length up to at least $20 \mathrm{~kb}$. The three normal groups have group-specific flanking sequences. The long normal group shares the flanking repeats with the expansions, suggesting that the expansions are derived from the long normal group. ${ }^{9}$ Expansions are caused by an increase in the copy number of the repeat motifs. Inverted AT-rich repeats form hairpin structures that may contribute to their further expansion. ${ }^{10}$ The expansion of AT-rich inverted repeats may generate perturbation of DNA replication. ${ }^{11}$ FRA10B is located in a transitional region between early and late zones of replication. Expanded alleles show a delayed replication pattern distal from the repeat, whereas proximally, no difference compared with normal alleles is seen. ${ }^{12}$

\section{MATERIALS AND METHODS}

All samples were analyzed as part of the Dutch TRIDENT study, which includes the analysis of aberrations other than trisomy 21, 18, and 13 (trisomies and subchromosomal aberrations of approximately $10 \mathrm{Mb}$ and more; sex chromosomal anomalies are not reported in the TRIDENT study). ${ }^{13,14}$ A license to perform this study was given by the Ministry of Health. All participants signed an informed consent form. Bioinformatic analysis was performed using WISECONDOR at default settings as described. ${ }^{15}$ Furthermore, adaptations to WISECONDOR were made to pinpoint the affected area more precisely. We increased the resolution by changing the bin size from $1 \mathrm{Mb}$ to $250 \mathrm{~kb}$. Speed and precision were improved by replacing LOESS GCcorrection with principal component analysis, where the principal component analysis transform was determined over the reference sample set. After mapping data to the principal component analysis dimensions (using the first three components), the original data were reconstructed and the difference between this reconstruction and the actual signal was taken as the read depth input per bin for WISECONDOR. The windowed $z$-score approach was replaced with a segmentation algorithm that focuses on finding the strongest Stouffer's $z$-scores over all possible windows per chromosome, allowing optimization of the call down to single bins. As these changes increase the number of tests per sample, the $z$-score threshold for significant aberrations had to increase as well. Instead of the usual threshold of 3 , the script determined the required $z$-score to be 4.8 for our purposes. All scripts are available from https://github.com/VUmcCGP/wisecondor.

Maternal lymphocytes were grown in Roswell Park Memorial Institute medium with or without adding $2 \mu \mathrm{M}$ BrdU for $24 \mathrm{~h}$. Fluorescence in situ hybridization (FISH) was performed with a probe for 10qter (GS-261-B16) and a control probe for 10pter (GS-23-B11). Probes were labeled in house using the BioPrime DNA Labeling System (Thermo Fisher Scientific, Waltham, MA). Array comparative genomic hybridization analysis was performed using either the $180 \mathrm{~K}$ SurePrint G3 Human CGH Microarray (Agilent Technologies, Santa Clara, CA) at the Academic Medical Center (AMC) or the Infinium CytoSNP-850k genotyping array (Illumina, San Diego, CA) at the Erasmus Medical Center (EMC).

\section{RESULTS}

In our original study, a total of 2,527 NIPS results were reported $^{14}$ In two cases, a terminal deletion of chromosome $10 \mathrm{q}$ was observed (a frequency of 1 in 1,263). The other six cases were added later. The original WISECONDOR results for the eight 10(q25 $\rightarrow$ ter) deletions are shown in Figure 1a. The enhanced NIPS pipeline was subsequently used for the three samples that were processed at VU University Medical Center (samples VUMC 1, AMC 1, and AMC 2). This highresolution analysis defined the individual start of the deletions as: between 113,250,000 and 135,000,000 with an effect size of $-3.07 \%$ for VUMC 1; between 113,000,000 and 135,000,000 with an effect size of $-4.54 \%$ for AMC 1; and between $112,750,000$ and $135,250,000$ with an effect size of $-4.39 \%$ for AMC 2 (GRCh37; Figure 1b). The effect size is the change in read depth compared with the expected number of reads, as determined by WISECONDOR.

To confirm the presence of the deletion in the fetus, routine array analysis was performed on DNA isolated from amniotic fluid in four cases (AMC 1, AMC 2, EMC 2, and EMC 3), but the deletion could not be confirmed in any of them. Placental biopsies were analyzed using FISH analysis with a 10qterspecific probe for AMC 1 (five biopsies from the cytotrophoblast layer and one from the mesenchymal core), or by single nucleotide polymorphism array for EMC 1 (one biopsy from the cytotrophoblast layer and one from the mesenchymal core) and EMC 2 and EMC 3 (both four biopsies from the cytotrophoblast layer and four from the mesenchymal core). Furthermore, the umbilical cord and blood were analyzed from EMC 1. The deletion was not found in any of these samples.

In an effort to explain the deletions, we realized that the breakpoints overlapped exactly with the FRA10E and FRA10B sites on 10q25.2. As the FRA10E site is a common fragile site present in all humans, we focused on FRA10B expansions as a possible cause of the deletions.

Culture of maternal blood lymphocytes with BrdU to induce a possible FRA10B fragile site showed a fragile site on chromosome 10 in all four of the cases tested: AMC $1(16 / 26$ cells; 61\%), AMC 2 (18/30 cells; 60\%), EMC 1 (17/30 cells; $57 \%$ ), and EMC 2 (14/50 cells; 28\%) (Figure 2a). We did not detect metaphases with an apparent deletion of 10(q25 $\rightarrow$ ter). When cultured according to standard procedures without BrdU, this fragile site was not expressed in blood lymphocytes. 

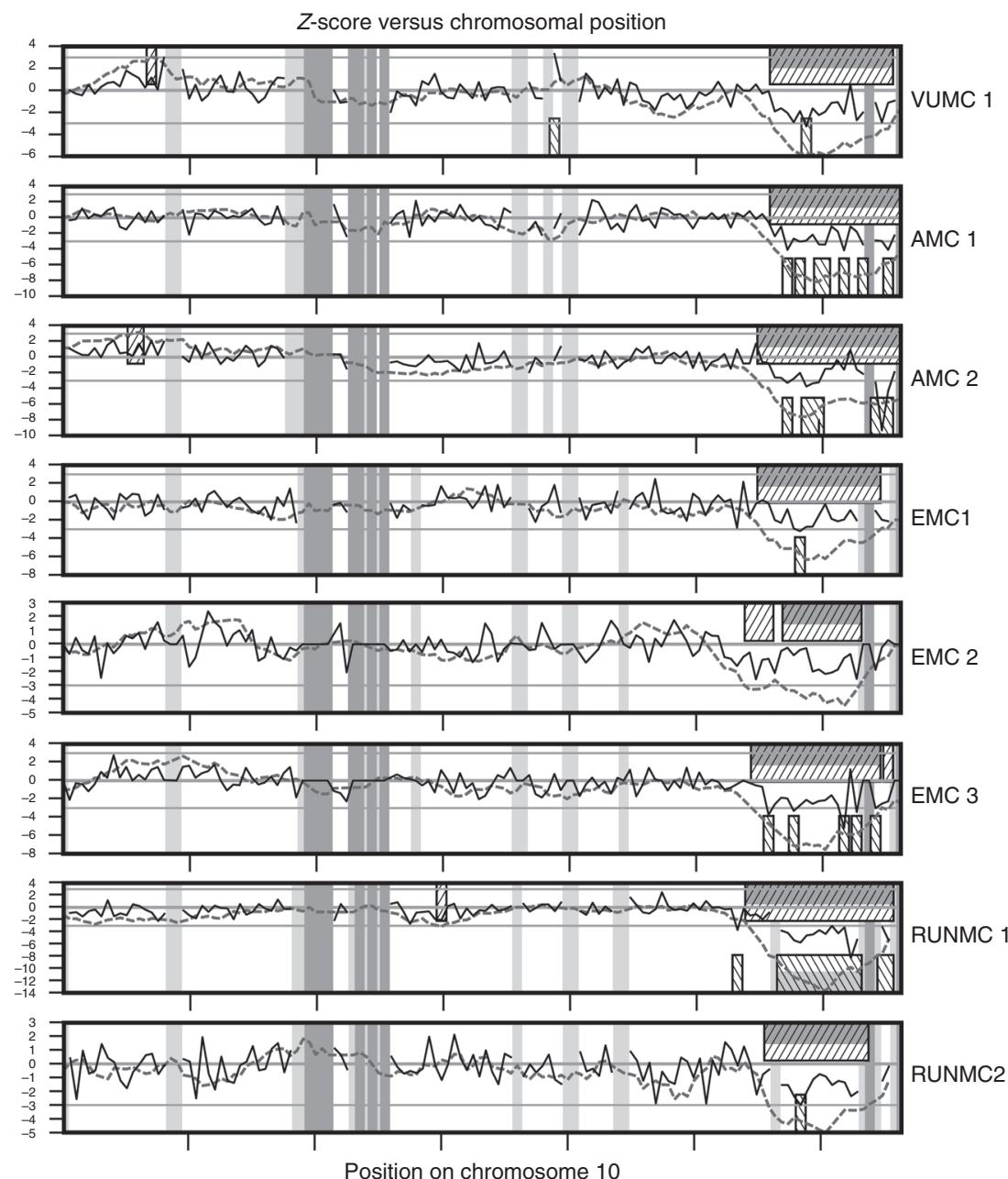

Position on chromosome 10

---. Z-score windowed method

_ Z-score individual bin method

एWID Detected deviation by windowed method

IIIII Called by windowed method
प107 Detected deviation by individual bin method МIIV Called by individual bin method

$\square$ Not enough reference bins available

$\square$ Unmappable region

b

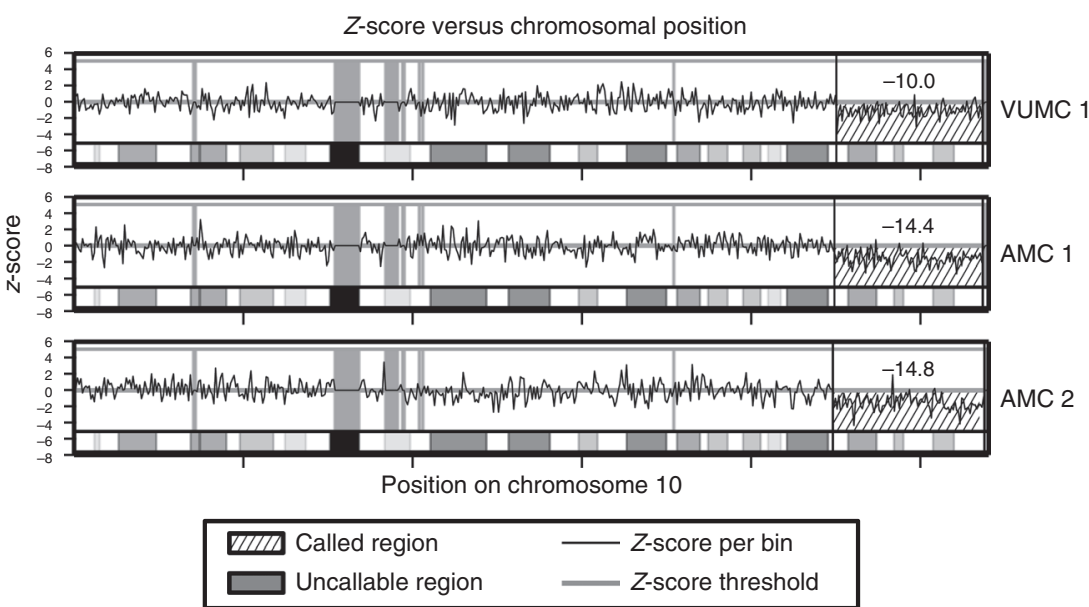


Additional FISH analysis on interphase nuclei of maternal blood lymphocytes cultured with BrdU in 2 cases showed a loss of signal for the $10 \mathrm{q}$ telomere in 7 of 203 nuclei in AMC 1 (3.4\%) and in 6 of 198 nuclei in AMC 2 (3.3\%), whereas a loss of signal for the 10p telomere was not observed in any of the nuclei. These FISH results suggested that carriers of FRA10B may exhibit low-level mosaicism for a 10q terminal deletion. To test this, in-depth array comparative genomic hybridization analysis was performed on DNA extracted from whole blood of the two AMC cases. This finding was confirmed in AMC 1, for which a mosaic deletion, distal from FRA10B, of approximately $11 \%$ was identified (Figure $2 \mathbf{b}$ ). No deletion could be detected in AMC 2, nor in EMC 2 and 3. As FRA10B is not associated with any clinical phenotype, we did not test the carrier status of the fetuses after birth.

\section{DISCUSSION}

Aberrations found by NIPS are not always of fetal origin, resulting in false-positive NIPS reports. Known examples of confounding factors are confined placental mosaics, maternal copy-number variants, and maternal malignancies. Knowledge of these factors is essential in proper NIPS analysis and counseling. Here, we describe an additional biological cause of discordant NIPS results. We tested and confirmed FRA10B expansions in four mothers where NIPS showed a 10q25-totelomere deletion. Initial FISH analysis showed the presence of a maternal low mosaic 10(q25 $\rightarrow$ ter) deletion in one case, probably as a consequence of the expanded fragile site. As the sensitivity of FISH analysis is insufficient to confirm or exclude very-low-grade mosaic deletions, we confirmed this finding using array analysis. As approximately $90 \%$ of the cellfree DNA tested during NIPS is maternal, this low maternal mosaic can be detected during NIPS analysis. The fact that the deletion was not seen in the peripheral blood of the other three cases of maternal FRA10B is probably due to mosaicism below the detection level of array. This does not exclude its presence in the maternal cell-free DNA fraction. FRA10B expression is induced by cellular stress. As cell-free DNA is derived from apoptotic cells it might even be enriched for the associated deletion. Assuming the occurrence rate for FRA10B is $2.5 \%$, as stated in previous work, the odds of finding four out of four individuals with FRA10B at random is $\sim 3.9 \times 10^{-7}\left(0.025^{4}\right)$, making our observation statistically significant at the usual significance threshold $(P<0.05)$.

Figure 1 Noninvasive prenatal testing results showing a deletion starting in 10q25 in eight pregnancies. (a) Initial WISECONDOR output. Data for each sample were analyzed using reference data for the center the sample was processed at. Samples are numbered for each center. (b) Plots showing the results of the enhanced WISECONDOR method applied to three samples with the 10q25.2 deletion. The $y$ axis shows $z$-scores for every bin shown on the $x$ axis. Numbers within the figures show the $z$-score of the called region. A chromosomal ideogram is visualized at the bottom of each plot. In all samples, the deletion starts at 10q25.2-the locus containing the FRA10B site (between 113,001,547 and 113,001,987). AMC, Academic Medical Center; EMC, Erasmus Medical Center; RUNMC, Radboud University Nijmegen Medical Centre; VUMC, VU University Medical Center Amsterdam.

a
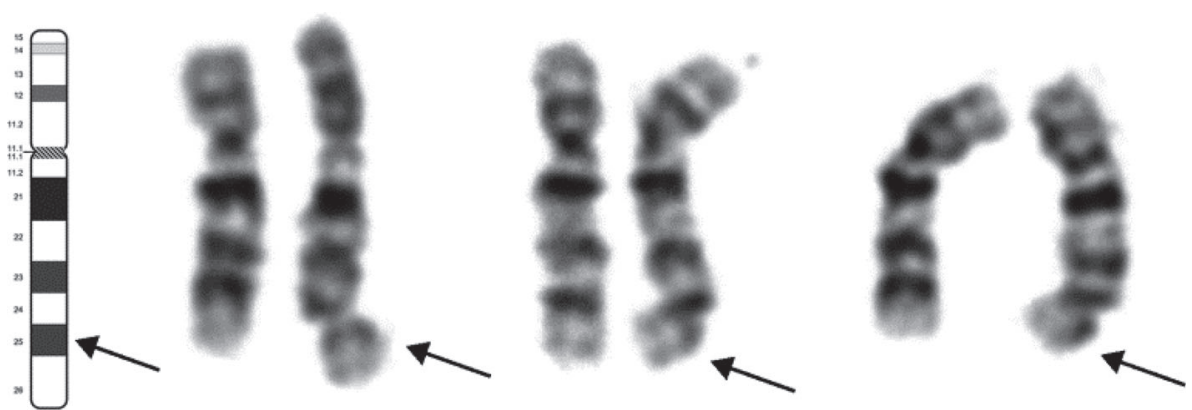

b

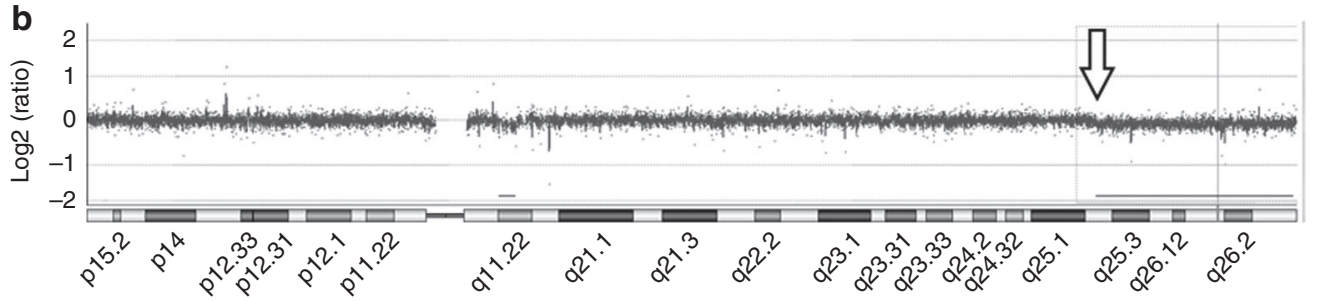

Figure 2 FRA10B expansions and mosaic maternal deletions. (a) GTG banding of chromosome 10 (case Academic Medical Center 1 (AMC 1)) after culture of blood lymphocytes in medium with bromodeoxyuridine to induce bromodeoxyuridine-sensitive fragile sites. The fragile site at $10 q 25$ is indicated by an arrow. (b) Array comparative genomic hybridization analysis of chromosome 10 of AMC 1 shows a low-grade mosaic loss of $10 \mathrm{q} 25 \rightarrow$ qter (breakpoint indicated by an arrow). The log ratio of this deleted region is -0.083 . 
There is ongoing debate as to whether NIPS should be targeted to trisomy 21,18 , and 13 alone, or whether it should be used as genome-wide screening to detect other chromosomal anomalies as well. Although the clear benefit of genome-wide testing is that more severe fetal anomalies will be detected, ${ }^{14}$ one of the arguments against it is that it will result in more false-positive results and therefore more invasive follow-up tests. However, many of these falsepositive results can easily be identified and explained without the need for invasive follow-up testing. In the case of maternal copy-number variants causing false-positive results, we have argued that, with the use of proper bioinformatical tools, it is easy to distinguish maternal copy-number variants from fetal trisomies. The same is true for maternal malignancies. ${ }^{16}$ Here, we identify another example of a relatively common cause of false-positive results that does not warrant follow-up by invasive testing. If a deletion starting exactly at FRA10B is found (Figure 1), it is highly likely that it is caused by a maternal mosaic deletion associated with a repeat expansion at this fragile site. This repeat expansion can be confirmed by maternal testing if preferred, although there is no known phenotype linked to this fragile site, even in homozygous carriers. The frequency at which we found the 10qter deletions is $\sim 1$ in 1,263 , which is much lower than the published carrier frequency of FRA10B expansions of 1 in $40 .{ }^{8}$ The most likely explanation for this discrepancy is that only in a small proportion of carrier women will FRA10B expansion result in a deletion at sufficiently high mosaic levels to be detected by routine NIPS analysis.

Several rare fragile sites are associated with mosaic deletions. ${ }^{6}$ We therefore investigated whether more recurrent deletions at fragile sites were observed. Thirty-one rare fragile sites have been described, ${ }^{7} 24$ of which are folate-sensitive sites consisting of CGG repeats such as the FRAXA repeat which is associated with fragile $\mathrm{X}$ syndrome. FRA10B belongs to the group of seven non-folate-sensitive sites consisting of AT-rich repeats. Although we did not detect recurrent deletions at any of these sites, we cannot exclude the possibility that in rare cases other fragile sites might explain false-positive NIPS results. Laboratories performing genomewide NIPS analysis should be aware of this. Overall, this finding increases the reliability and health benefits obtained through NIPS. It also proves that classical cytogenetic knowledge is still very important for the proper interpretation of NIPS results, as the last scientific paper on FRA10B dates from 2003. ${ }^{17}$

\section{ACKNOWLEDGMENTS}

The authors thank the Dutch NIPT consortium and all pregnant women participating in the TRIDENT studies.

\section{DISCLOSURE}

The authors declare no conflict of interest.

\section{REFERENCES}

1. Zhang $H, G a o Y$, Jiang $F$, et al. Non-invasive prenatal testing for trisomies 21, 18 and 13: clinical experience from 146,958 pregnancies. Ultrasound Obstet Gynecol 2015;45:530-538

2. Wang L, Meng Q, Tang X, et al. Maternal mosaicism of sex chromosome causes discordant sex chromosomal aneuploidies associated with noninvasive prenatal testing. Taiwan J Obstet Gynecol 2015;54: 527-531

3. Snyder MW, Simmons LE, Kitzman JO, et al. Copy-number variation and false positive prenatal aneuploidy screening results. $N$ Engl J Med 2015;372:1639-1645

4. Bianchi DW, Chudova D, Sehnert AJ, et al. Noninvasive prenatal testing and incidental detection of occult maternal malignancies. JAMA 2015;314:162-169

5. Gromminger S, Yagmur E, Erkan S, et al. Fetal aneuploidy detection by cell-free DNA sequencing for multiple pregnancies and quality issues with vanishing twins. J Clin Med 2014;3:679-692

6. Glover TW, Stein CK Chromosome breakage and recombination at fragile sites. Am J Hum Genet 1988;43:265-273

7. Lukusa T, Fryns JP Human chromosome fragility. Biochim Biophys Acta 2008;1779:3-16

8. Sutherland GR Heritable fragile sites on human chromosomes. IX. Population cytogenetics and segregation analysis of the BrdU-requiring fragile site at 10q25. Am J Hum Genet 1982;34:753-756

9. Hewett DR, Handt O, Hobson L, et al. FRA10B structure reveals common elements in repeat expansion and chromosomal fragile site genesis. $\mathrm{Mol}$ Cell 1998;1:773-781

10. Yu S, Mangelsdorf M, Hewett $D$, et al. Human chromosomal fragile site FRA16B is an amplified AT-rich minisatellite repeat. Cell 1997;88: 367-374

11. Handt O, Sutherland GR, Richards RI Fragile sites and minisatellite repeat instability. Mol Genet Metab 2000;70:99-105

12. Handt $O$, Baker E, Dayan S, et al. Analysis of replication timing at the FRA10B and FRA16B fragile site loci. Chrom Res 2000;8:677-688

13. Oepkes D, Page-Christiaens GC, Bax CJ, et al. Trial by Dutch laboratories for evaluation of non-invasive prenatal testing. Part I-clinical impact. Prenat Diagn 2016;36:1083-1090

14. Van Opstal D, Van Maarle MC, Lichtenbelt K, et al. Origin and clinical relevance of chromosomal aberrations other than the common trisomies detected by genome-wide NIPS: results of the TRIDENT study. Genet Med; e-pub ahead of print 2 October 2017

15. Straver R, Sistermans EA, Holstege H, Visser A, Oudejans CBM, Reinders MJT WISECONDOR: detection of fetal aberrations from shallow sequencing maternal plasma based on a within-sample comparison scheme. Nucleic Acids Res 2014;42:e31

16. Sistermans $E$, Straver $\mathrm{R}$, Faas $B H$ Maternal malignancies detected with noninvasive prenatal testing. JAMA 2015;314:2192

17. Zlotorynski E, Rahat A, Skaug J, et al. Molecular basis for expression of common and rare fragile sites. Mol Cell Biol 2003;23:7143-7151 